\title{
Survey and analysis of the prophylactic and medical activity of the elderly and the old people.
}

\author{
Tanya Paskaleva1 ${ }^{1}$, Biyanka Tornyova $^{2}$, Snezhana Dragusheva $^{3}$, Penka Petleshkova ${ }^{4}$ \\ ${ }^{1}$ Department of Health Care, Trakia University of Stara Zagora, Bulgaria \\ ${ }^{2}$ Department of Health Care Management, Medical University of Plovdiv, Plovdiv, Bulgaria \\ ${ }^{3}$ Department of Nursing Care, Faculty of Public Health, Medical University, Plovdiv, Bulgaria \\ ${ }^{4}$ Department of Obstetrics and Gynecology, Medical University Plovdiv, Bulgaria
}

\begin{abstract}
Health prophylaxis is a strategic priority in every health care system. This research aims at surveying the prophylactic and medical activity of the elderly and the old. An anonymous survey of 340 people aged 60 and over was conducted in "Haskovo Hospital" for a period of one year. The collected data provide grounds for concluding that health prophylaxis as a direct reflection of health education, and health awareness is not adequately utilized or offered medical activity, which is fundamental to achieving better health, preventing diseases and premature ageing. The percentage of elderly and old people who go through regular preventive medical check-ups is highly unsatisfactory. This puts forward significant problems connected with the organization of prophylaxis, the lack of health motivation, negligence towards prophylaxis, passive and irresponsible attitude.
\end{abstract}

Keywords: Health prophylaxis, Prophylactic activities, The elderly and the old.

Accepted on April 9, 2019

\section{Introduction}

The process of ageing of the population is becoming one of the most significant social transformations of the $21^{\text {st }}$ century. The following are but a few of the main points emphasized in the National strategies: development of policies for active ageing, adapting the social and health care services system, and improving the quality of life of senior citizens. Health prophylaxis is also of high priority, as its role and importance are beyond any doubt $[1,2]$. The attitude to health and the way of life are vital for reducing the incidence of diseases/ morbidity/and improving the quality of life of the elderly and the old [3-6]. The general strategies aimed at attaining a beneficial effect on chronic diseases among senior citizens include: regular, systematic medication therapy, regular physical rehabilitation courses, hygiene and diet-related measures, a balanced diet, and active exercise routines designed to optimize the way of life [7].

In recent years, it has been identified that there is a lack of effective actions aimed at all levels of prophylaxis: Preprimary-aimed at preventing the onset and progress of the disease, and identifying social, economic and behavior models that contribute to enhancing the risk of a disease. Primaryaimed at preventing the disease from developing through a control of the causes and risk factors. Secondary-aimed at curbing the progress of an already manifested disease through early diagnostics, treatment, and prophylaxis of recurrences. Tertiary prophylaxis-aimed at improving the quality of life and preventing complications and disabilities in cases of already developed diseases [8].

In addition of regular prophylactic procedures, higher awareness, motivation and health culture of the population are needed. For the successful outcome of any treatment, it is essential that patient should accept and stick to it strictly. The improved consent and appropriate changes in patients' behavior are beneficial to all parties involved in the health care system-medical specialists, patients, pharmaceutical companies, health insurance funds [9].

Old age manifests itself with specific decrease of the functional abilities of the human organism. This distinguishes a specific age group from the rest of the population, a group which causes numerous specific medical and social problems that are especially difficult to solve [10]. The treatment and physical rehabilitation of such patients call for profound medical and social procedures and actions leading to an improvement of the quality of life of the patient and enhancing/facilitating emotional and mental adaptation [11]. The principles of the therapeutic approach - the treatment plan/scheme - are that it should be independent and tailored to the status of the respective patient [12]. The early detection of a disease, its diagnostics, staging and the identification of its form, are of 
vital importance. Therapy has to follow the strict sequence of the rehabilitation therapy algorithms whilst taking into consideration the individual specifics of each patient, approaching and applying kinesitherapeutic programs in a complex and comprehensive way. In modern physical rehabilitation, the holistic approach is mandatory; it is based on comprehensiveness: cognitive ability (orientation, attention span, memory, the ability to collaborate in the process of the medical interventions); range of movement (active and passive); muscle strength (weakness), locomotor deficiency (if any); coordination; agility; resilience [13].

Preventive activities are mainly within the scope of responsibilities of the General practitioner. Annual prophylactic medical check-ups are mandatory, and they are regulated by Ordinance no.39 of 2004, with subsequent supplementations and amendments [14]. The medical activities stipulated for the elderly include: one annual mandatory full medical check-up, consisting of a clinical examination, arterial blood pressure, ECG, full blood count, blood sugar, urine test albumin and sediment. People with chronic diseases are treated as out-patients subjected to regular monitoring, depending on their condition. Targeted preventive examinations of people over 65 are another important aspect, and they are mainly designed to facilitate the early detection and diagnostics of oncological and other diseases.

Information provided by the National Health Fund shows that $38 \%$ of the population of our country underwent mandatory prophylactic medical check-ups in 2018 [15]. Figure 1 illustrates the results connected with the frequency and attendance of prophylactic check-ups.

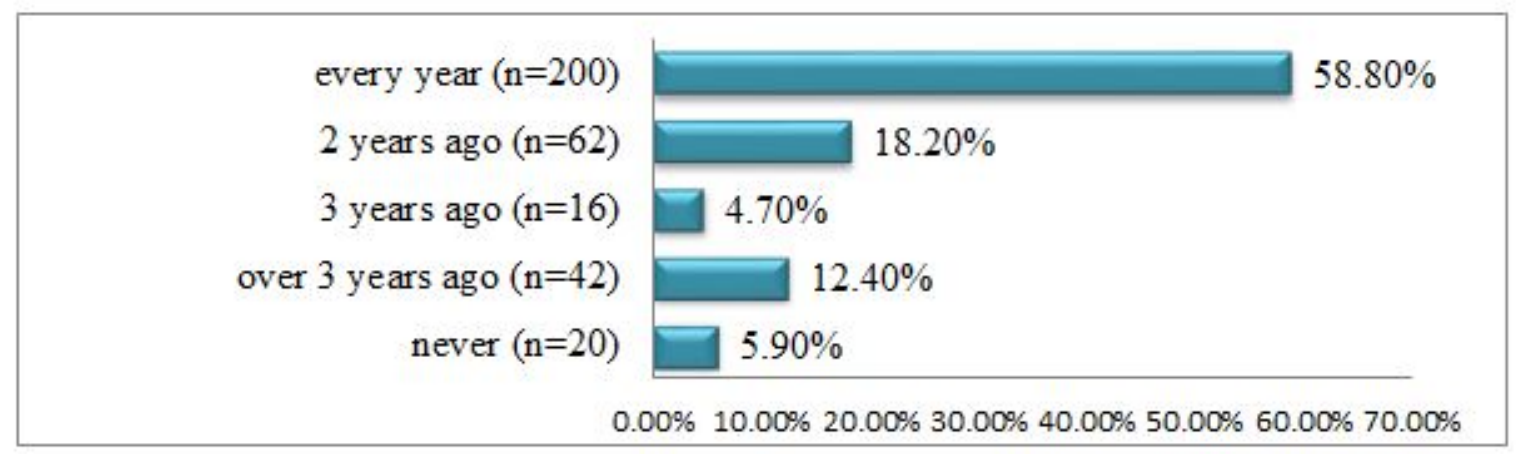

Figure 1. Frequency of prophylactic medical check-ups.

The investigated connection between prophylactic check-ups and the factorial variable of "place of residence" shows that city dwellers $(67.5 \%)$ undergo prophylactic check-ups every year, while those living in the country - mainly once every two years $(23.4 \%)$ and once every three or more years $(17.2 \%)$ $\mathrm{p}=0,001 \quad\left(\chi^{2}=18.57\right)$. Shashwat Nagar et al. in a survey conducted in the rural regions of India in 2013, established that nearly half of the elderly population suffers from one or more diseases, and most of those people did not visit medical centres for prophylactic check-ups and did not look for medical help at all [16]. These findings show a fact that has an adverse effect on the provision of prophylactic care for the elderly.

\section{Aim}

The aim is to survey and analyze the prophylactic and medical activity of elderly and old people.

\section{Subjects and Methods}

An anonymous survey was carried out among people age 60 and over. The study was conducted in patients in several different clinical departments of "Haskovo Hospital" in Haskovo, Bulgaria. Participants in the study were selected by age and opportunity for adequate participation. Criteria for inclusion are: age over 60 years and able to respond adequately to the questionnaire. The study included all patients over the age of 60 , able to respond adequately to the questionnaire that is prepared for this survey.

\section{Applied methods}

Descriptive statistics of quantitatively measurable values. The results are presented as mean, standard deviation (Std Deviation) and standard error mean (Std error mean); dispersion analysis (one way ANOVA). Alternative analysis was applied in processing quality parameters/attributes, and the result is presented with a relative share; non-parametric analysis-consent criterion of Pierson $\left(\chi^{2}-\mathrm{x}\right.$ square $)$ in testing hypotheses for statistically significant connection between the investigated factorial and resulting parameters; correlation analysis of Spearman-to evaluate the level of the dependence between the investigated parameters; graphic analysis-to illustrate processes and phenomena, as well as to illustrate certain regularities and dependencies, the options provided by graphic analysis were used. $\mathrm{P}<0.05$ was assumed/accepted as the level of significance of the zero hypothesis.

\section{Results and Discussion}

The study included a total of 304 patients over the age of 65 . The respondents were interviewed during a hospital stay in clinical wards in Haskovo Hospital. A questionnaire was prepared specifically for the purposes of the study and all respondents signed an informed consent to participate. 
Analyzing their age distribution, we established that over half of them, $50.6 \%$, were in the $60-70$ age group. In terms of sex, $58.2 \%$ of the surveyed were women, and $41.8 \%$-men. In terms of family status, the group of the married ones was the biggest (at $53.8 \%$ ), followed by that of the single people-widow/ widowers (at $30.9 \%$ ). Of the people taking part in our survey, $62.4 \%$ live in the city, as compared to $37.6 \%$ permanently residing in the country. The survey of people in places of various distance to the city was also focused on the opportunity for access to medical help-primary and specialized.

The main source of income for $87.3 \%$ of the surveyed is their pension, which is highly inadequate, so only $20.9 \%$ of them can make ends meet on the income they receive. Ageing is connected greater and greater expenses, since most diseases people develop then are chronic and require continuous, and in many cases 'life-long, treatment, sometimes involving costly medical procedures. A significant proportion of the survey are actually below the poverty line-57.1\%. This economic instability, insecurity and vulnerability often put the elderly in difficult situations connected with their physical survival and deprivations, which, in turn, constitute a risk factor with potential health implications.

The findings regarding the awareness of the respondents of the prophylactic check-ups show that most of the surveyed answered "Yes"-69.7\%, whereas 30.3\% state they have not been informed. The analysis of the connection between awareness and the factorial variable of "education" established that people with a lower level of education (40.3\%) are more often unaware of the annual prophylactic check-ups $\mathrm{p}=0.001$ $\left(\chi^{2}=18.93\right)$. We also established that elderly people who live alone $(42.9 \%)$ are not well informed of scheduled prophylactic activities $p=0.01\left(\chi^{2}=11.39\right)$. Therefore, it is clear why those living alone undergo annual prophylactic medical check-ups more rarely, $(48.6 \%) \mathrm{p}=0,039\left(\chi^{2}=21.88\right)$.

To all respondents who gave a positive answer and were fully aware of the regular prophylactic check-ups (69.7\%), the logical follow-up question was how they had been informed. According to $48.5 \%$ of the surveyed state they are invited to a prophylactic check-up during a visit to their GP, which, in turn, indicates they visit their doctor on account of a health problem more often. $24.3 \%$ of the surveyed stated they had been informed by telephone, and $23.4 \%$ - with a notice posted on the info board at the GP's practice (Figure 2).

Over half of the respondents- $58.8 \%$, state that they undergo the mandatory prophylactic check-ups every year, have a positive attitude towards health prophylaxis and believe its role is significant. This result may be attributed to the fact that the elderly belong to a generation that is characterized by a strong sense of responsibility, strictness and even pedantry. On the other hand, to old people of both sexes health is the dominant value. This privileged position of health in the hierarchy of values presupposes a higher level of personal responsibility for health and a positive health conduct. The results of the rest of the respondents are alarming; they manifest partial or total negligence, and their relative share is significant $-41.2 \%$.
In the course of our survey, it was important to us to identify the causes for not undergoing prophylactic medical check-ups. These are facts and circumstances that cause a negative reflex, and serve as a motivation basis for health behaviour with undesirable consequences.

As clearly shown in Figure 3, the main reasons are lack of information-30.3\%, and difficulties in reaching to the GP's place-27.6\%. The distance from the respective village of the elderly to the GP's practice also causes serious inconveniences and difficulties. $11.8 \%$ of the surveyed state that the GP doctor comes to their village on certain days and for a few hours only. A substantial part of the Bulgarian population lives in places where there are no GP's practices. Those are usually isolated, sparsely populated places: In such cases the options the elderly can choose from are: selecting a practice in another town/ village, or opting for a GP who comes to their village only on certain days, for a few hours. In both cases, the inconveniences, the difficulties and the increased health risk are at the expense of all those people living in unattractive regions".

A quarter of the surveyed (25\%) state the main reason for not going to prophylactic check-ups is the long time they spend waiting on the queue in front of the GP's cabinet. While we do take into account the objective factors hampering people's access to their GP, but we would also like to emphasize the significant role of personal responsibility and motivation regarding health, and in many of the cases people demonstrate a passive, irresponsible attitude by neglecting prophylactic check-ups.

The findings of the survey concerning out-patients' visits to a doctor show that in the past six months $68.2 \%$ of the respondents requested medical care and help, mostly on account of a chronic condition $(30.9 \%)$, or an acute disease $(17.1 \%)$. It is of note that the higher their disability is, the more often such patients have sought medical help in the past 6 months $(\mathrm{F}=3.90)$.

The findings regarding the frequency of seeking medical help by "sex" show that women have requested medical aid more often in the past 6 months $\mathrm{P}=0,01(\mathrm{u}=2.61)$. To a great extent this relates to the fact that women, as a whole, were established to be suffering from a greater number of diseases, which presupposes higher levels of health needs and a greater level of consumption of healthcare services (Table 1).

More than $1 / 2$ of all respondents seek medical help when their health deteriorates, which may be attributed to the fact that a great proportion of the elderly rarely complain about their health (Figure 4) In their desire not to be a burden to and not to worry their families and friends, they sometimes exhibit excessive tolerance, and hence problems connected with untimely sought medical help, a more advanced stage of the condition, and a higher risk of complications. According to the results from the survey, dwellers of rural areas seek medical help more often when their health seriously deteriorates $(58.6 \%) \mathrm{p}=0,005(\chi 2=12.80)$. This fact can also be accounted for by the lack of medical centres in cases of need for medical 
help in the country, which may pose serious inconveniences and difficulties. In this way, people with reduced virtually have no access to what they need.

In this survey it was established that mostly married people look for medical help and care when the first symptoms of a condition appear (32.2\%), followed by those living with their children $(35.9 \%)$, whereas the ones who live alone usually do not seek medical help $(29.5 \%) \quad p=0.019 \quad(\chi 2=19.80)$. These results connect well to some other results showing that elderly people living on their own resort to self-treatment more often $(47.6 \%) \mathrm{p}=0.016\left(\chi^{2}=10.380\right)$, as compared to those living with a spouse $(29.0 \%)$, with family $(30.8 \%)$ and with their children $(33.3 \%)$

The higher the education of the respondents, the more frequent their visits to the GP. Immediately upon detecting the first symptoms of a disease, old people with college education $(46.4 \%)$ and those with a university degree $(60 \%)$ seek medical help. In addition to the higher qualification, income is another factor which has a direct effect on people's attitude; old people from the higher-income group tend to seek medical health at onset of the condition $(41.8 \%) \mathrm{p}=0,001\left(\chi^{2}=24.06\right)$.
As regards employment, respondents still in employment seeking medical help at the very onset of a condition comprise $58.3 \%$, those who are old-age pensioners $-54.1 \%$, and retired people with disabilities - only when their condition deteriorates, at $65.5 \%$.

The results regarding the frequency of seeking medical care by "ethnic groups" show that over $1 / 3$ of the surveyed Roma $(38.9 \%)$ state that they "do not always" opt for medical care. We attribute these results of high-risk behavior to the lack of finances needed for consumer fees, transport to the medical center, as well as medication. That is why self-treatment is a common practice among the Roma ethnos [17-20].

Table 1. Frequency of seeking medical help by "sex".

\begin{tabular}{lllll}
\hline Sex & $\mathbf{N}$ & Mean & Std. Deviation & Std. Error Mean \\
\hline Men & 91 & 2.10 & 1.300 & 0.136 \\
\hline Women & 141 & 2.65 & 1.732 & 0.146 \\
\hline
\end{tabular}

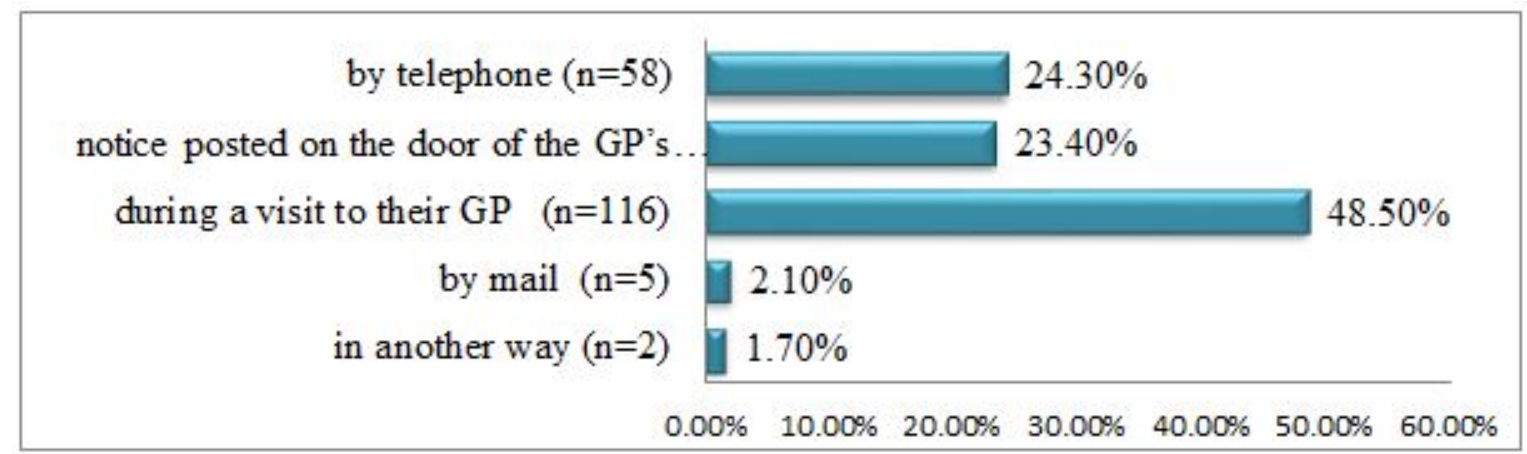

Figure 2. Ways and means of accessing information about prophylactic examinations.

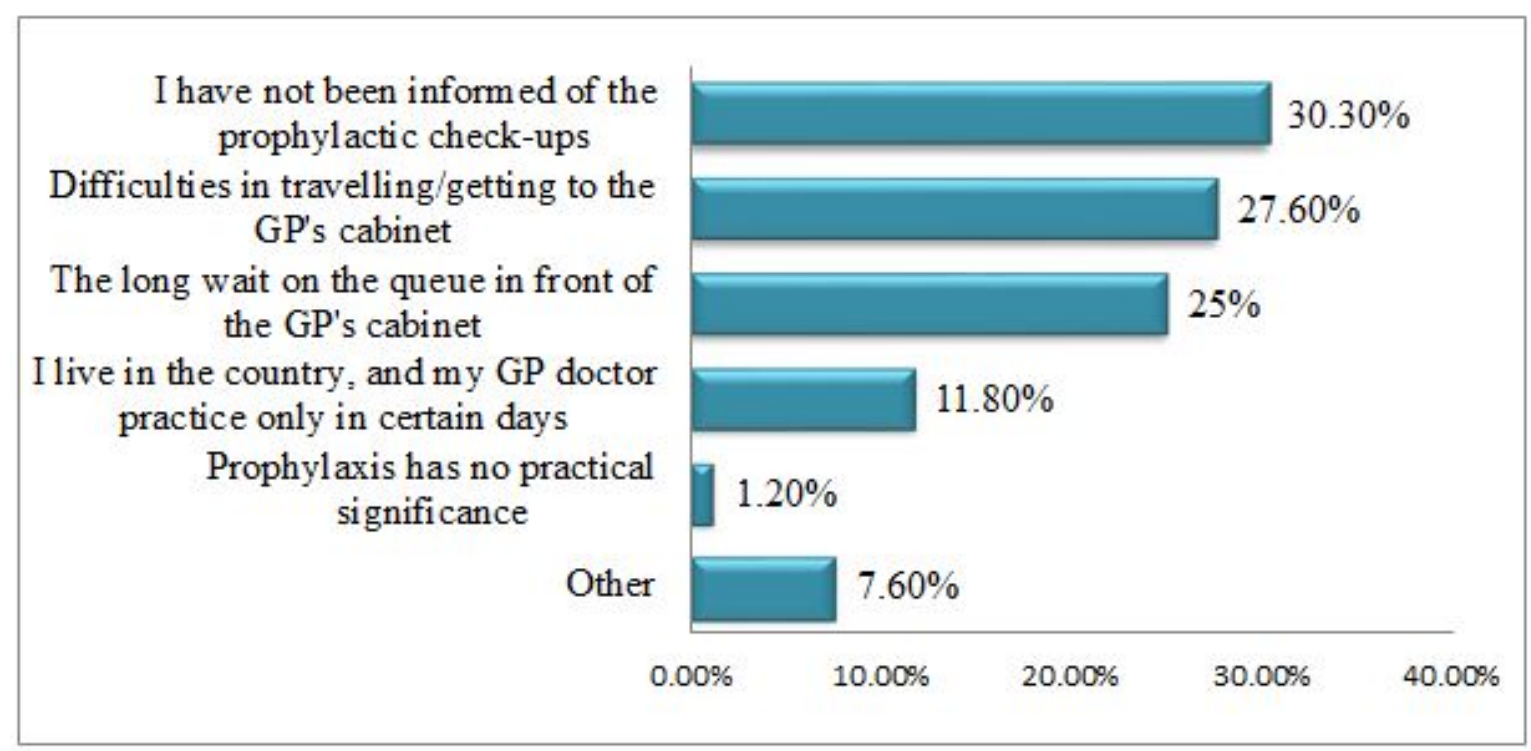

Figure 3. Reasons for not undergoing prophylactic check-ups. 


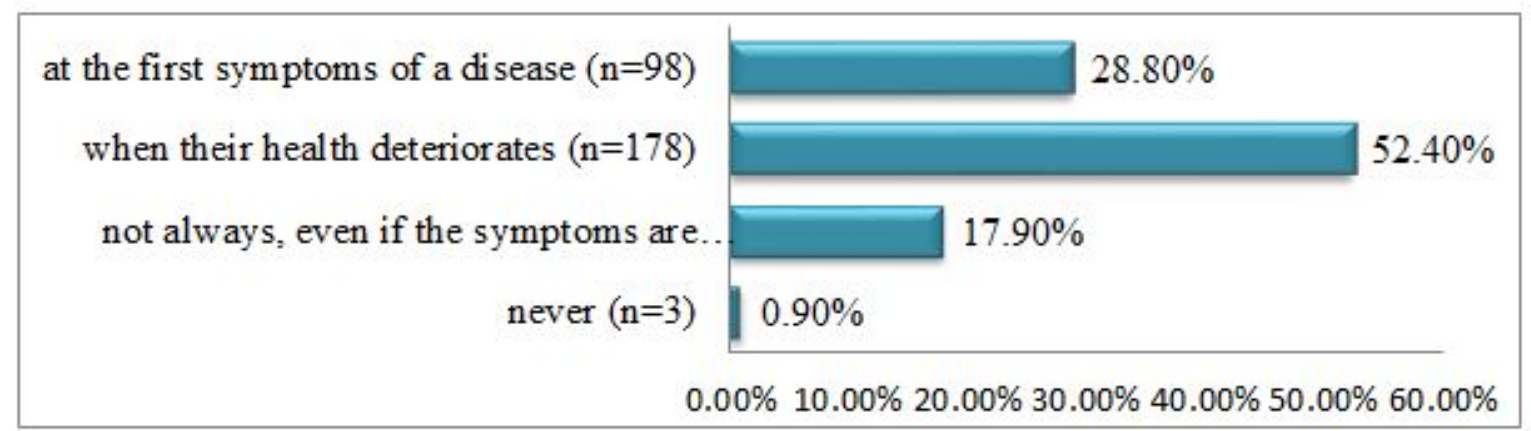

Figure 4. Frequency of seeking medical care and help.

\section{Key Findings}

Health prophylaxis as a response related to health education and health awareness is not adequately utilized, provided medical activity, which is fundamental and vital for achieving good health and preventing from diseases and premature ageing.

The percentage of elderly and old people who regularly undergo the mandatory annual prophylactic check-ups is unsatisfactory. A significant proportion of the surveyed $(41.2 \%)$ demonstrate partial or complete negligence to prophylaxis, and hence to their health.

The key factors for not attending prophylactic check-ups are found to be: lack of information $-30.3 \%$, and difficulties in in reaching to the GP's cabinet $-27.6 \%$.

At the first signs of a disease, the ones who immediately seek medical help are married couples $(32.2 \%)$, followed by elderly and old people living with their offspring (35.9\%). The elderly with higher education who seek medical help are $60 \%$ of the surveyed people and those with higher income are $41.8 \%$. These findings are indicative of the fact that social and economic conditions have a significant effect on the individual health-related risk factors. A timely visit to a medical professional at the first symptoms of a disease may prevent a series of hospitalizations and the use of healthcare resources.

Over $1 / 2$ of all respondents seek medical care when their health deteriorates. With elderly people, the facts connected with the higher incidence of complications should not be underestimated.

We may consider the strengths of the study that the modifying factors relevant to prophylaxis and medical activities of the elderly have been studied. But the weak side of the study is that it has not been studied all the factors affecting older people in their approach to health services. As well as the fact that the study is based on the subjective assessment of the participants. It would be good to make a larger retrospective and prospective study on this topic.

\section{Conclusion}

In the modern conditions of a dynamically changing world, in which demographic ageing of the population is escalating at an unprecedented rate, and its repercussions affect all spheres of social life, the problems connected with elderly people will become more and more pressing in modern societies.

The main burden of the care for the elderly is on primary medical care. Healthcare systems should focus their efforts on providing a continuum of care: prophylaxis, early diagnostics and treatment, rehabilitation and palliative care. The implementation of early diagnostic methods allows for detecting the process of premature ageing in its early stage, and the medical control of the risk factors and prompt corrective actions in the event of a devitalized way of life serve as a guarantee for an active, meaningful, full-value old age.

\section{References}

1. National Strategy for Demographic Development of the Population in the Republic of Bulgaria 2012-2030; 99-110.

2. Bulgarian National Health Strategy 2020, 31-35.

3. Fries JF, Carpo L. Vitality and aging: Implications of the rectangular curve. New York: WH Freeman 1981.

4. Rowe JW, Kahn RL. Human aging: Usual and successful. Science 1987; 237:143-149.

5. Stults BM. Preventive health care and the elderly. West J Am Med 1984 ;41:832-845.

6. Jagger C, Matthews R, Matthews F, Robinson T, Robine JM, Brayne C. The burden of diseases on disability-free life expectancy in later life. J Gerontol A Biol Sci Med Sci 2007; 62: 408-414.

7. Geuorguiev St, Kafalova D, Madzharov V, Staynova R, Stoimenova A, Lebanova H, Mihaylova A, Geuorguieva PE. Pharmaceutical care in adult patients. Pharma Care Guide 2019; 10.

8. Vodenicharov TS, Popova S, Mutafova M, Shipkovenska E. Social medicine. GorexPres Sofia 2013; 417-426.

9. St. Geuorguiev, Kafalova D, Madzharov V, Staynova R, Stoimenova A, Lebanova H, Mihaylova A, Geuorguieva EP. Strategies to improve patient compliance. Pharma Care Guide 2019; 62.

10. Visev K, Borisova B, Viseva M. Quality management of primary health care in elderly people. Medical Meridians 2018; 1:20-25. 
11. Kasnakova $\mathrm{P}$, Ivanova $\mathrm{S}$, Ivanov $\mathrm{K}$, Gueorguieva $\mathrm{PE}$, Gueorguiev S, Madzharov V, Mihaylova A, Petleshkova P. Conservative therapy options for the treatment of coxarthrosis in the early stage of the condition. BMR 2018; 29:2997-3001.

12. Kasnakova P, Mihaylova A, Petleshkova P. Comprehensive rehabilitation of herniated disc in the lumbar section of the spine. BMR 2018; 29:3002-3005.

13. Kasnakova P, Obreshkova D, Petkova V, Atanasov P. Physical rehabilitation procedures and holistic approaches to the treatment of some neurodegenerative diseases. WJPPS 2017; 6 .

14. Ordinance No. 39 of 16 November 2004 for preventive examinations and dispensarisation.

15. https://www.nhif.bg/

16. Nagar S, Nagar N, Nagar S, Mahyawanshi D, Katha GP. Impact of social factors on health practices of the elderly: An analytical study in rural Surendranagar. IJRMS 2013; 1:116-122.

17. Kane RL, Kane RA, Arnold SB. Prevention and the elderly: risk factors. Health Serv Res 1985; 19:945-1006.
18. Lubben JE, Weiler PG, Chi I. Health practices of the elderly Poor. Am J Public Health 1989; 79:731-734.

19. Leinonen R, Heikkinen E, Jylha M. Predictors of decline in self-assessments of health among older people-- a 5year longitudinal study. Soc Sci Med 2001; 52:1329-1341.

20. Begum MS. Geriatric health problems and health care seeking practice among elderly people attending one selected geriatric hospital. J Physiol Pharmacol 2007; 23:20-24.

\section{*Correspondence to}

\section{Penka Petleshkova}

Department of Obstetrics and Gynecology

Medical University Plovdiv

Plovdiv

Bulgaria 